Table DR1. Walvis Ridge Nd isotope data generated in this study.

\title{
Hole 1262A
}

$\begin{array}{cccc}\text { Depth }(\mathbf{m c d}) & \text { Age }(\mathrm{Ma}) & { }^{143} \mathrm{Nd} \mathbf{I}^{144} \mathrm{Nd} & \mathcal{E}_{\mathrm{Nd}}(\mathbf{t}) \\ 55.63 & 16.53 & 0.512088 \pm 13 & -11.24 \pm 0.26 \\ 56.33 & 17.08 & 0.512125 \pm 08 & -10.50 \pm 0.16 \\ 56.33 & 17.08 & 0.512113 \pm 06 & -10.73 \pm 0.12 \\ 56.34 & 17.09 & 0.512156 \pm 07 & -9.90 \pm 0.14 \\ 59.42 & 19.54 & 0.512137 \pm 08 & -10.20 \pm 0.16 \\ 60.92 & 20.73 & 0.512117 \pm 09 & -10.56 \pm 0.18 \\ 62.42 & 21.92 & 0.512116 \pm 07 & -10.55 \pm 0.14 \\ 63.92 & 23.12 & 0.512158 \pm 08 & -9.71 \pm 0.16 \\ 65.42 & 24.31 & 0.512136 \pm 07 & -10.10 \pm 0.14 \\ 66.92 & 25.50 & 0.512190 \pm 08 & -9.02 \pm 0.16 \\ 68.89 & 27.07 & 0.512147 \pm 07 & -9.82 \pm 0.14 \\ 70.39 & 28.26 & 0.512160 \pm 07 & -9.53 \pm 0.14 \\ 72.56 & 29.98 & 0.512167 \pm 08 & -9.36 \pm 0.16 \\ 74.89 & 31.84 & 0.512196 \pm 07 & -8.74 \pm 0.14 \\ 76.39 & 32.95 & 0.512187 \pm 07 & -8.89 \pm 0.14 \\ 81.04 & 35.39 & 0.512167 \pm 07 & -9.22 \pm 0.14 \\ 82.46 & 36.75 & 0.512337 \pm 08 & -5.87 \pm 0.16 \\ 84.53 & 38.72 & 0.512285 \pm 08 & -6.84 \pm 0.16 \\ 85.06 & 39.22 & 0.512167 \pm 07 & -9.13 \pm 0.14 \\ 85.67 & 39.81 & 0.512198 \pm 08 & -8.50 \pm 0.16 \\ 87.1 & 41.17 & 0.512144 \pm 07 & -9.52 \pm 0.14 \\ 91.71 & 50.24 & 0.512145 \pm 04 & -9.28 \pm 0.08 \\ 93.21 & 50.44 & 0.512122 \pm 06 & -9.72 \pm 0.12 \\ 105.78 & 52.12 & 0.512110 \pm 04 & -9.91 \pm 0.08 \\ 115.22 & 53.12 & 0.512114 \pm 08 & -9.81 \pm 0.16 \\ 125.28 & 53.65 & 0.512165 \pm 07 & -8.79 \pm 0.14 \\ 146.885 & 55.39 & 0.512200 \pm 07 & -8.08 \pm 0.14 \\ 157.325 & 56.49 & 0.512146 \pm 07 & -9.11 \pm 0.14 \\ 167.625 & 57.19 & 0.512194 \pm 07 & -8.15 \pm 0.14 \\ 176.555 & 58.36 & 0.512230 \pm 07 & -7.42 \pm 0.14\end{array}$

$\begin{array}{cccc}\begin{array}{c}\text { Hole 1263A } \\ \text { Depth (mcd) }\end{array} & \text { Age (Ma) } & { }^{143} \mathbf{N d} / /^{144} \mathrm{Nd} & \varepsilon_{\mathrm{Nd}}(\mathrm{t}) \\ 29.3 & 25.22 & 0.512114 \pm 07 & -10.52 \pm 0.14 \\ 51.72 & 29.68 & 0.512169 \pm 07 & -9.32 \pm 0.14\end{array}$




$\begin{array}{cccc}63.08 & 31.44 & 0.512189 \pm 08 & -8.89 \pm 0.16 \\ 74.18 & 31.65 & 0.512170 \pm 05 & -9.26 \pm 0.10 \\ 84.74 & 31.85 & 0.512204 \pm 07 & -8.58 \pm 0.14 \\ 94.26 & 32.95 & 0.512205 \pm 07 & -8.55 \pm 0.14 \\ 105.14 & 36.01 & 0.512219 \pm 08 & -8.19 \pm 0.16 \\ 114.8 & 36.38 & 0.512194 \pm 07 & -8.67 \pm 0.14 \\ 138.12 & 39.37 & 0.512173 \pm 07 & -9.00 \pm 0.14 \\ 150.49 & 41.06 & 0.512176 \pm 11 & -8.89 \pm 0.22 \\ 161.36 & 43.54 & 0.512124 \pm 09 & -9.86 \pm 0.18 \\ 171.99 & 44.55 & 0.512131 \pm 07 & -9.69 \pm 0.14 \\ 182.41 & 45.62 & 0.512158 \pm 08 & -9.15 \pm 0.16 \\ 193.44 & 46.77 & 0.512150 \pm 07 & -9.28 \pm 0.14 \\ 205.24 & 47.63 & 0.512128 \pm 08 & -9.67 \pm 0.16 \\ 217.31 & 48.38 & 0.512131 \pm 06 & -9.60 \pm 0.12 \\ 228.55 & 48.55 & 0.512123 \pm 06 & -9.75 \pm 0.12 \\ 238.51 & 50.07 & 0.512277 \pm 06 & -6.71 \pm 0.12 \\ 246.92 & 50.12 & 0.512134 \pm 08 & -9.49 \pm 0.16 \\ 263.62 & 51.61 & 0.512113 \pm 08 & -9.87 \pm 0.16 \\ 276.3 & 52.99 & 0.512162 \pm 05 & -8.88 \pm 0.10\end{array}$

\section{Hole 1264A}

$\begin{array}{cccc}\text { Depth }(\mathbf{m c d}) & \text { Age }(\mathbf{M a}) & { }^{143} \mathbf{N d} /^{144} \mathbf{N d} & \mathcal{E}_{\mathbf{N d}}(\mathbf{t}) \\ 209.36 & 17.69 & 0.512143 \pm 14 & -10.13 \pm 0.28 \\ 209.36 & 17.69 & 0.512127 \pm 09 & -10.44 \pm 0.18 \\ 219.92 & 19.82 & 0.512132 \pm 10 & -10.29 \pm 0.20 \\ 228.94 & 21.00 & 0.512135 \pm 07 & -10.21 \pm 0.14 \\ 240.81 & 22.11 & 0.512142 \pm 10 & -10.05 \pm 0.20 \\ 251.87 & 22.73 & 0.512184 \pm 08 & -9.21 \pm 0.16 \\ 261.25 & 24.21 & 0.512155 \pm 07 & -9.74 \pm 0.14 \\ 273.12 & 26.96 & 0.512145 \pm 08 & -9.86 \pm 0.16 \\ 300.48 & 29.41 & 0.512170 \pm 08 & -9.32 \pm 0.16\end{array}$

Teeth and fragments of fish debris were handpicked from the $>63$ micron size-fraction of washed (disaggregated) samples from discrete 1 to $2 \mathrm{~cm}$ intervals of ODP Holes 1262A, 1263A, and 1264A. All depths are giving in meters composite depth (mcd), which refers to meters below sea floor in the stratigraphically spliced section. Multiple teeth/fragments were used in each analysis (in general, from 5 to 15 teeth/fragments per sample, depending on size and availability). Samples were then cleaned using an established reductive/oxidative cleaning protocol (Boyle, 
1981; Boyle and Keigwin, 1985). Samples were analyzed as $\mathrm{NdO}^{+}$using a multi-collector Micromass Sector 54 at the radiogenic isotope facility at the University of North Carolina Chapel Hill. Monitor peak $\left({ }^{144} \mathrm{Nd}^{16} \mathrm{O}\right)$ beams of $\sim 0.5$ to 1 volt were achieved by introducing pure oxygen into the source via a leak valve. External analytical precision based upon replicate analysis of the international standard $\mathrm{JNd}_{\mathrm{i}}$ (Tanaka et al., 2000) yielded $0.512111 \pm 0.000010$ $(n=30)$ which is calibrated relative to the La Jolla standard (0.511858) as 0.512116. Reported errors are within-run $2 \sigma$ values, which corresponds to a minimum uncertainty of \pm 0.28 epsilon units when combined with the external precision. The procedural blank is $\sim 15 \mathrm{pg}$ and is considered negligible. Replicate analyses of several samples yielded Nd isotope values within error limits.

Because fish debris record the rare earth elemental composition of seawater, it is necessary to analyze the Sm content in order to correct for any in situ production of ${ }^{143} \mathrm{Nd}$ (being the daughter of ${ }^{147} \mathrm{Sm}$ ). We applied a mean ${ }^{147} \mathrm{Sm} /{ }^{144} \mathrm{Nd}$ value of 0.131 (derived from analyses of Walvis Ridge DSDP Site 527 (Thomas et al., 2003) to calculate $\varepsilon_{\mathrm{Nd}}(\mathrm{t}$ ) values.

\section{Supplementary References}

Boyle, E.A., 1981, Cadmium, zinc, copper, and barium in foraminifera tests: Earth and Planetary Science Letters, v. 53, p. 11-35.

Boyle, E.A. and Keigwin, L.D., 1985, Comparison of Atlantic and Pacific paleochemical records for the last 250,000 years: changes in deep ocean circulation and chemical inventories: Earth and Planetary Science Letters, v. 76, p. 135-50. 